\title{
Soluble myeloid triggering receptor expressed on myeloid cell 1 might have more diagnostic value for bacterial ascites than C-reactive protein
}

https://doi.org/10.1515/biol-2018-0054

Received June 27, 2018; accepted September 29, 2018

\begin{abstract}
The timely and accurate diagnosis of ascites is of great significance for early treatment and prognostication. This study explored the value of soluble myeloid triggering receptor expressed on myeloid cell 1 (sTREM-1) and C-reactive protein (CRP) for assessing ascites. A total of 133 patients with ascites who received treatment at the Affiliated Hospital of Taishan Medical University between September 2015 and September 2017 were retrospectively analyzed. The ascites in 22, 45, 33 and 33 patients were tuberculous, bacterial, tumorous, and transudative, respectively. Healthy volunteers $(n=30)$ who received a health examination at the same hospital during the same period constituted the control group. Before treatment, both ascitic STREM-1 and CRP showed significant differences among the ascites subgroups $(\mathrm{P}<0.001)$, with the highest levels in the bacterial subgroup. Serum sTREM-1 and CRP also showed significant differences among the groups. A correlation analysis showed a positive correlation between STREM- 1 and CRP. ROC curves of the bacterial subgroup showed that when the optimal cutoff point was set to 20.2, the sensitivity, specificity, positive predictive value, and negative predictive value of the serum sTREM-1 index were 0.933, 0.955, 0.914, and 0.965, respectively. STREM-1 may provide more diagnostic value than CRP for the diagnosis of bacterial ascites.
\end{abstract}

Keywords: soluble myeloid triggering receptor expressed on myeloid cell 1 (sTREM-1), ascites, condition judgment, ELISA, immunoturbidimetry

\footnotetext{
*Corresponding author: Qiang Guo, Department of Infectious Diseases, Affiliated Hospital of Taishan Medical University, Tai'an 271000, China, E-mail: guoqcn18@163.com Chuanqin Xu, Chao Sun, Yubao Zhao, Department of Infectious Diseases, Affiliated Hospital of Taishan Medical University, Tai'an 271000, China

Weifu Zhang, Department of Public Health, Affiliated Hospital of Taishan Medical University, Tai'an 271000, China
}

\section{Introduction}

Under normal conditions, a small amount of liquid exists in the human abdominal cavity $(<200 \mathrm{ml})$ and provides lubrication for intestinal peristalsis, and the volume of intraperitoneal fluid might increase under pathological conditions. When the amount surpasses $200 \mathrm{ml}$, ascites occurs. Approximately 38-52\% of ascites are malignant [1], and according to their etiology and characteristics, ascites can be divided into various types, including bacterial, tuberculous, tumorous, and transudative ascites. The characteristics of a patient's ascites have a direct influence on his/her treatment and prognosis evaluation. However, the differentiation of ascites has long been a challenge for clinical and laboratory diagnosis, and therefore, the identification of biomarkers for ascites differentiation remains urgent.

To date, different methods have been proposed for the differentiation of ascites. The preferred method of diagnosis is exfoliative cytology. However, due to various factors, this method has a positive rate of only $30 \%$ [2]. Conditions of inflammation, infection, myocardial infarction and tumorigenesis induce significant changes in the plasma concentrations of a certain class of proteins, which are collectively referred to as acute-phase reaction proteins (APRPs). Among different APRPs, C-reactive protein (CRP) has a very low serum concentration in the healthy population but a significantly increased concentration after bacterial infection and tissue damage. For this reason, it has been considered the most valuable bioactive indicator for ascites differentiation and has been applied in clinical practice [3, 4]. However, under some conditions, such as obesity, sleep disorder, depression, chronic fatigue, aging, physical activity, radiation therapy and smoking, an increase in CRP might also occur, and the CRP levels may not accurately reflect the specificity of individuals with these conditions. Therefore, the identification of a more convenient and more reliable bioactive indicator remains necessary. 
Myeloid triggering receptor expressed on myeloid cell 1 (TREM-1) serves as an inflammatory receptor that is primarily expressed by myeloid cells (e.g., neutrophils and mononuclear macrophages) [5-7]. It exists in two forms in organisms: as a membrane protein on the surface of mononuclear macrophages and as a soluble myeloid triggering receptor expressed on myeloid cell 1 (sTREM1) in body fluids. STREM-1 can activate the expression of a variety of inflammatory factors [8] and thus serves as an important diagnostic marker of inflammation as well as a new early warning indicator of infectious diseases. Its upregulation can be detected in pathogen culture solution, peritoneal lavage fluid, and tissue samples from a site of infection [6]. In bacterial pneumonia, the STREM-1 level in pulmonary tissue is significantly higher than that in serum, and STREM-1 can serve as an independent diagnostic marker of bacterial pneumonia [9]. As a newly discovered important diagnostic marker of inflammatory disease, sTREM-1 holds great potential for clinical applications [10], but to the best of our knowledge, previous studies have not determined whether STREM-1 can serve as a new biomarker for ascites differentiation.

Based on the aforementioned findings, we explored the diagnostic value of STREM-1 for the differentiation of ascites type in this study. The results might provide a new method for the accurate diagnosis of ascites and deepen the understanding of this disease condition.

\section{Materials and methods}

\subsection{Study subjects}

A total of 133 patients with ascites who received treatment between September 2015 and September 2017 at the Affiliated Hospital of Taishan Medical University were consecutively enrolled in this retrospective cohort study. The diagnostic criteria were based on the literature [11, 12], and the patients included 81 males and 52 females. Their ages ranged from 33 to 86 years, with an average of $54.2 \pm 3.8$ years, and 22, 45, 33 and 33 patients had tuberculous ascites (Mycobacterium tuberculosis based on ascitic cultures, typical clinical symptoms, ascites absorption after normal antituberculosis treatment), bacterial ascites (bacterial culture, bacterial discovery according to microscopy, and ascites disappearance after antibacterial+dieresis treatment), tumorous ascites (ascitescytology, B-ultrasound, and computed tomography (CT)), and transudative ascites (hyponatremia transudate according ascites routine examination and biochemistry), respectively. The following inclusion criteria were used:
1) ascites according to B-ultrasound or CT, with a depth $\geq 5$ $\mathrm{cm}$ and nonencapsulated effusion; 2) no severe heart, liver, lung, or kidney disorders; 3) between 18 years and 90 years of age; and 4) informed consent. Patients meeting any of the following were excluded from the study: 1) intestinal obstruction or abdominal adhesions; 2) presence of cardiac, liver, kidney, lung or cerebrovascular diseases; 3) poor compliance or refusal to cooperate; 4) hemorrhagic ascites due to trauma; 5) insufficient amount of ascites extracted; and 6) tuberculous ascites combined with bacterial ascites.

Thirty healthy volunteers who received a health examination at the same hospital during the same period constituted the control group. These include 17 males and 13 females and had an average age of $54.1 \pm 4.3$ years.

After confirmation of the ascites type, the tuberculous group was administered isoniazid $(0.3 \mathrm{~g}$, qd), rifampicin (0.45 g, qd) and pyrazinamide ( $0.5 \mathrm{~g}$, tid), the bacterial group received cefotaxime (4 g, bid) or ofloxacin $(500 \mathrm{mg}$, qd), the tumorous group was treated with fluorouracil (0.25-0.5 g per day or every two days; 5-10 g within a curative course) or carboplatin $\left(300-400 \mathrm{mg} / \mathrm{m}^{2}\right)$, and the transudative group was administered human serum albumin (10 g, qd). In addition, all four groups received furosemide ( $40 \mathrm{mg}$, qd). The treatment course was $14 \mathrm{~d}$.

Informed consent: Informed consent has been obtained from all individuals included in this study.

Ethical approval: The research related to human use has been complied with all the relevant national regulations, institutional policies and in accordance the tenets of the Helsinki Declaration, and has been approved by the ethics committee of the Affiliated Hospital of Taishan Medical University.

\subsection{Sample collection}

At 3 p.m., $10 \mathrm{ml}$ of ascitic fluid was extracted from each patient using conventional abdominal paracentesis. The sample was placed in an anticoagulation tube containing $0.1 \mathrm{ml}$ of sodium citrate. At 7:00 a.m., $3 \mathrm{ml}$ of fasting venous blood was collected from the patients and the controls into an anticoagulation tube containing $0.1 \mathrm{ml}$ of sodium citrate. After 10-20 min of mixing, the samples were centrifuged at $3000 \mathrm{r} / \mathrm{min}(\mathrm{R}=18 \mathrm{~cm})$ for $10 \mathrm{~min}$. The supernatants were then collected and stored at $-80^{\circ} \mathrm{C}$.

Fourteen days after treatment, fasting venous blood samples were obtained from all the treatment groups. 


\subsection{ELISA}

The sTREM-1 level was detected by ELISA. Each sample was thawed at room temperature, and after buffer ( $\mathrm{pH}$ 7.4) was added, and the solution was shaken at a constant temperature for $60 \mathrm{~min}$. The sample solution was then centrifuged at $10,000 \mathrm{r} / \mathrm{min}$ at $4^{\circ} \mathrm{C}$ for $10 \mathrm{~min}$ to allow the precipitation of bacterial and cellular residues to the bottom of the tube. The supernatant was separated into microcentrifugation tubes. The sTREM-1 level was detected in strict accordance with the procedures indicated in the kit (R\&D, Minneapolis, MN, USA; coefficient of variation: within batch, $\leq 4.0 \%$; batch-to-batch, $\leq 7.0 \%$ ).

The detection was performed by experts with clinical laboratory qualification based on the single-blind method, and thus, the experts were not aware of the grouping outcomes.

\subsection{Immunoturbidimetry}

The ascitic and serum CRP levels were determined by immunoturbidimetry. Whole blood or ascites was extracted, allowed to coagulate and centrifuged at 3500 $\mathrm{r} / \mathrm{min}$. The serum/supernatant was detected using a 7600-110E automatic analyzer (HITACHI, Japan) with a kit purchased from Leadman (Beijing, China), which had an intra-batch coefficient of variation of $\leq 5.0 \%$ and a batch-tobatch coefficient of variation of $\leq 8.0 \%$. The experimental procedures were performed in accordance with the instructions provided by the manufacturer of the kit.

\subsection{Statistical analysis}

The data were processed using SAS 8.2 statistical software.

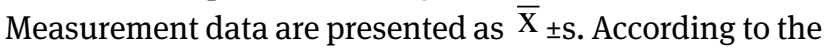
normality of the data and homogeneity of variance, a paired t-test or analysis of variance was performed; otherwise, the Kruskal Wallis test was performed. The correlation analysis was performed using Spearman's correlation method. Enumerated data are presented as percentages. A chi-square test was used for comparisons between groups. Receiver operating characteristic curves were plotted to assess the diagnostic precision of the indices. The Youden index was used for critical value calculation, and the sensitivity, specificity, positive predictive value and negative predictive value of each diagnostic index were also calculated. $\mathrm{P}<0.05$ was considered to indicate statistical significance.

\section{Results}

\subsection{General data}

No significant differences in sex or age were observed among the ascites and control groups ( $\mathrm{P}>0.05$; Table 1$)$.

Ascitic and serum STREM-1 and CRP levels of the different types of ascites

The ascitic and serum STREM-1 and CRP levels before treatment showed significant differences among the different ascites groups $(\mathrm{P}<0.001)$. The highest ascitic and serum STREM-1 levels were observed in the bacterial group, followed by the tuberculous, timorous, and transudative groups. Similarly, the highest serum sTREM-1 levels were observed in the bacterial group, followed by the tuberculous, tumorous, transudative, and control groups (Table 1).

\subsection{Correlation analysis of ascitic and serum sTREM-1 and CRP levels before treatment}

Scatter diagrams were plotted (Figure 1). Because ascitic and serum STREM-1 and CRP level data were not normally

Table 1. General data and monitoring indices of the different ascites groups.

\begin{tabular}{|c|c|c|c|c|c|c|c|c|}
\hline & Index & Bacterial & Tuberculous & Tumorous & Transudative & Healthy control & $F^{\prime} X^{2}$ & $\mathbf{P}$ \\
\hline \multirow[t]{2}{*}{ Gender } & Male & $27(27.6 \%)$ & $13(13.3 \%)$ & $20(20.4 \%)$ & $21(21.4 \%)$ & $17(17.3 \%)$ & 0.333 & $0.988 a$ \\
\hline & Female & $18(27.7 \%)$ & $9(13.8 \%)$ & $13(20.0 \%)$ & $12(18.5 \%)$ & $13(20.0 \%)$ & & \\
\hline Age (years) & & $54.56 \pm 4.2$ & $55.9 \pm 3.4$ & $53.1 \pm 4.1$ & $53.7 \pm 3.2$ & $54.1 \pm 4.3$ & 7.239 & $0.124 b$ \\
\hline \multirow{2}{*}{$\begin{array}{l}\text { Pretreatment } \\
\text { ascitic fluid }\end{array}$} & sTREM-1 & $468.3 \pm 61.4$ & $310.1 \pm 39.3$ & $425.9 \pm 40.8$ & $163.2 \pm 34.5$ & - & 103.5 & $<.001 \mathrm{~b}$ \\
\hline & CRP & $2.6 \pm 1.4$ & $2.2 \pm 1.6$ & $1.1 \pm 0.8$ & $0.9 \pm 0.6$ & - & 43.4 & $<.001 \mathrm{~b}$ \\
\hline \multirow{2}{*}{$\begin{array}{l}\text { Pre-treatment } \\
\text { serum }\end{array}$} & sTREM-1 & $38.2 \pm 11.4$ & $15.6 \pm 6.4$ & $14.8 \pm 2.7$ & $12.1 \pm 4.6$ & $11.8 \pm 3.2$ & 98.2 & $<.001 \mathrm{~b}$ \\
\hline & CRP & $15.1 \pm 4.4$ & $12.6 \pm 3.1$ & $3.6 \pm 1.4$ & $2.1 \pm 1.2$ & $1.8 \pm 1.1$ & 127.0 & $<.001 \mathrm{~b}$ \\
\hline
\end{tabular}

Notes: $a, X^{2}$ test; $b$, Kruskal Wallis test. 
distributed, Spearman's correlation analysis was performed. The outcomes showed that before treatment, the ascitic sTREM-1 level was positively correlated with the ascitic CRP the $(\mathrm{r}=0.666, \mathrm{P}<0.001)$, and the serum $\mathrm{STREM}-1$ levels was positively correlated with the ascitic CRP level $(\mathrm{r}=0.802, \mathrm{P}<0.001)$.

\subsection{ROC curve analysis}

For the bacterial group, the areas under the ascitic sTREM-1 and CRP ROC curves (AUC) were both between 0.7 and 0.9, and those with regard to serum sTREM-1 and CRP were above 0.9 (all $\mathrm{P}<0.001$ ). Pairwise comparisons showed that the AUC of serum STREM-1 was significantly greater than any of the other three AUCs (Figure 2). When the optimal cutoff point was set to 20.2, the sensitivity, specificity, positive predictive value, and negative predictive value of the serum sTREM-1 index were 0.933, 0.955, 0.914, and 0.965, respectively. Serum STREM-1 was found to be more accurate for diagnosing bacterial ascites than any of the other indices (Table 2).

In the other groups, CRP did not exhibit a higher diagnostic value than sTREM-1 (Table 3).
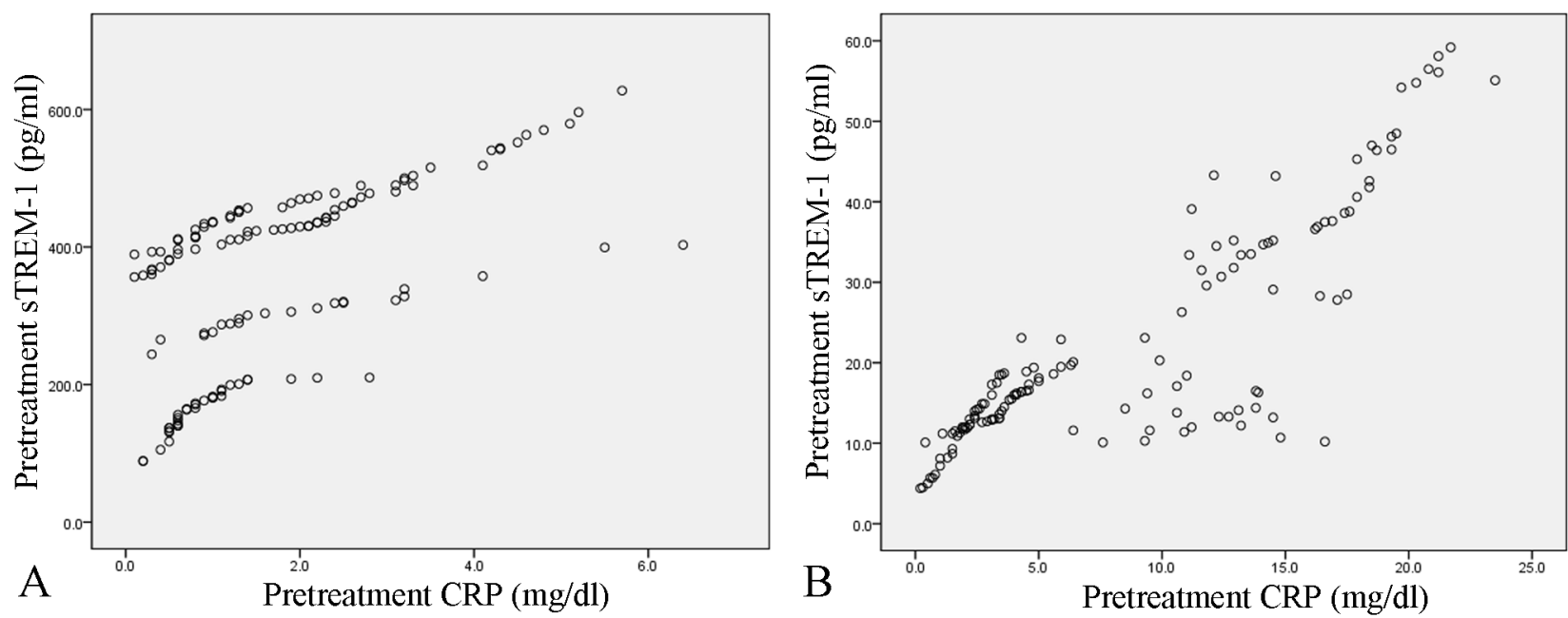

Figure 1. Scatter diagrams of ascitic and serum STREM-1 and CRP levels before treatment. A, ascitic fluid. B, serum.

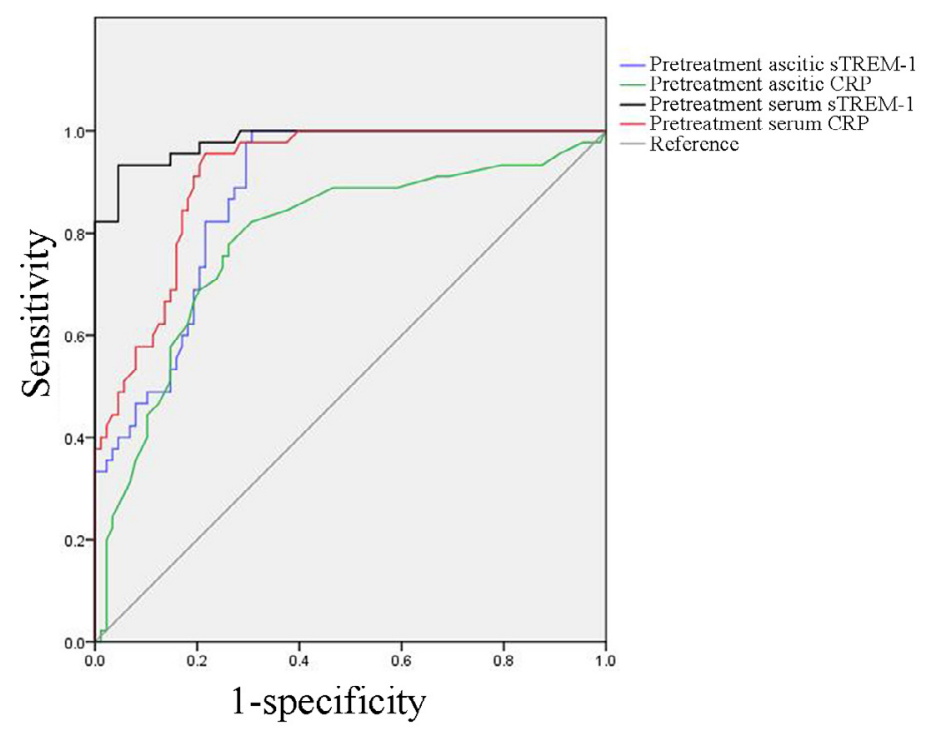

Figure 2. ROC curves of STREM-1 and CRP in ascitic fluid and serum for the diagnosis of bacterial ascites. 


\subsection{Serum STREM-1 and CRP levels before and after treatment}

The serum STREM- 1 and CRP levels in the bacterial group after treatment were significantly decreased compared with those before treatment $(\mathrm{P}<0.001$; Table 4). However, in the other groups, no significant differences in the STREM-1 and CRP levels were observed between before and after treatment.

\section{Discussion}

CRP is an APRP that is primarily synthesized and secreted by hepatic cells and regulated and induced by a variety of factors, such as IL-6, IL- 1 and TNF- $\alpha$. Because CRP can be secreted in high quantities by hepatic cells and enters the circulation within 12-48 $\mathrm{h}$ after its induction by damage and various inflammatory stimuli, it has a certain degree of reference value for differentiating the nature of ascites

Table 2. Characteristic analysis of STREM-1 and CRP in ascitic fluid and serum samples from the bacterial group.

\begin{tabular}{|c|c|c|c|c|c|c|c|c|c|}
\hline Index & Cutoff point & Sensitivity & Specificity & $\begin{array}{l}\text { Youden } \\
\text { index }\end{array}$ & PPV & NPV & AUC & $95 \% \mathrm{Cl}$ & $\mathbf{P}$ \\
\hline Ascitic sTREM1 & 385.4 & 1 & 0.693 & 0.693 & 0.625 & 1 & 0.877 & $(0.821,0.933)$ & $<0.001$ \\
\hline Ascitic CRP & 1.45 & 0.778 & 0.739 & 0.517 & 0.604 & 0.867 & 0.787 & $(0.700,0.873)$ & $<0.001$ \\
\hline Serum sTREM1 & 20.2 & 0.933 & 0.955 & 0.888 & 0.914 & 0.965 & 0.981 & $(0.962,0.999)$ & $<0.001$ \\
\hline Serum CRP & 8.9 & 0.956 & 0.784 & 0.74 & 0.694 & 0.972 & 0.914 & $(0.869,0.959)$ & $<0.001$ \\
\hline
\end{tabular}

Notes: PPV, positive predictive value; NPV, negative predictive value; $\mathrm{AUC}$, area under the curve; $\mathrm{Cl}$, confidence interval

Table 3. Characteristics of ascitic and serum STREM-1 and CRP in the samples from the four groups.

\begin{tabular}{lllll}
\hline Group & Index & AUC & $95 \% \mathrm{Cl}$ & $P$ \\
\hline Bacterial & Ascitic STREM1 & 0.877 & $(0.821,0.933)$ & $<0.001$ \\
& Ascitic CRP & 0.787 & $(0.700,0.873)$ & $<0.001$ \\
Serum STREM1 & 0.981 & $(0.962,0.999)$ & $<0.001$ \\
Tuberculous & Serum CRP & 0.914 & $(0.869,0.959)$ & $<0.001$ \\
& Ascitic STREM1 & 0.309 & $(0.224,0.394)$ & 0.005 \\
& Ascitic CRP & 0.618 & $(0.496,0.741)$ & 0.080 \\
Tumorous & Serum STREM1 & 0.322 & $(0.219,0.424)$ & 0.008 \\
& Serum CRP & 0.728 & $(0.648,0.808)$ & 0.001 \\
& Ascitic STREM1 & 0.689 & $(0.603,0.775)$ & 0.001 \\
Transudative & Ascitic CRP & 0.326 & $(0.229,0.424)$ & 0.003 \\
& Serum STREM1 & 0.351 & $(0.262,0.440)$ & 0.011 \\
& Serum CRP & 0.265 & $(0.184,0.347)$ & 0.000 \\
& Ascitic STREM1 & 0.000 & $(0.000,0.000)$ & $<0.001$ \\
& Ascitic CRP & 0.242 & $(0.159,0.325)$ & $<0.001$ \\
& Serum STREM1 & 0.204 & $(0.123,0.284)$ & $<0.001$ \\
\end{tabular}

Notes: AUC, area under the curve; $\mathrm{Cl}$, confidence interval.

Table 4. Serum sTREM-1 $(\mathrm{pg} / \mathrm{ml})$ and CRP $(\mathrm{mg} / \mathrm{dl})$ levels before treatment and after the $14-\mathrm{d}$ treatment.

\begin{tabular}{|c|c|c|c|c|c|c|c|c|}
\hline \multirow{2}{*}{ Group } & \multicolumn{2}{|c|}{ Bacterial $(n=45)$} & \multicolumn{2}{|c|}{ Tuberculous $(n=22)$} & \multicolumn{2}{|c|}{ Tumorous $(n=33)$} & \multicolumn{2}{|c|}{ Transudative $(\mathrm{n}=33)$} \\
\hline & STREM-1 & CRP & sTREM-1 & CRP & STREM-1 & CRP & sTREM-1 & CRP \\
\hline Pretreatment & $38.2 \pm 11.4$ & $15.1 \pm 4.4$ & $15.6 \pm 6.4$ & $12.6 \pm 3.1$ & $14.8 \pm 2.7$ & $3.6 \pm 1.4$ & $12.1 \pm 4.6$ & $2.1 \pm 1.2$ \\
\hline Post-treatment & $13.6 \pm 7.2$ & $3.5 \pm 1.3$ & $15.4 \pm 6.3$ & $12.1 \pm 3.8$ & $14.5 \pm 3.6$ & $3.6 \pm 2.0$ & $11.9 \pm 4.6$ & $2.0 \pm 1.1$ \\
\hline$t / Z$ & 30.973 & 24.538 & -1.007 & 1.142 & 0.812 & 0.350 & 1.463 & 1.466 \\
\hline$P$ & $<0.001 c$ & $<0.001 c$ & $0.314 d$ & $0.266 c$ & $0.423 c$ & $0.729 c$ & $0.153 c$ & $0.152 c$ \\
\hline
\end{tabular}

Notes: c, pairwise $t$ test; $d$, Wilcoxon signed rank test. 
$[13,14]$. However, CRP can be influenced by several factors and diseases and therefore exhibits low sensitivity and specificity [4]. TREM-1 is an inflammatory receptor that has attracted great attention from scholars in recent years [5-7]. In patients with acute cholangitis, TREM-1 expression is upregulated, and TREM-1 exhibits higher sensitivity and specificity to this condition than other clinical inflammatory markers [15]. In acute pancreatitis, the serum sTREM-1 level is noticeably increased and positively correlated with the severity of the disease [16]. Considering the role played by TREM-1 in amplifying inflammation, detecting the serum level of TREM-1 is a promising method for effectively evaluating inflammation as well as disease prognosis. In this study, we explored the diagnostic value of sTREM-1, one of the important existing forms of TREM-1, for the differentiation of different ascites types.

Our study showed that before treatment, the ascitic sTREM-1 and CRP levels in all the ascites groups were significantly higher than those in the healthy controls $(\mathrm{P}<0.001)$, with the highest levels in the bacterial group, followed by the tuberculous, tumorous and transudative groups. The serum STREM- 1 and CRP levels in the different ascites groups were also significantly higher than those in the healthy controls $(\mathrm{P}<0.001)$, with the highest levels in the bacterial group, followed by the tuberculous, tumorous and transudative groups. A correlation analysis showed a positive correlation between the sTREM-1 and CRP levels in both ascitic liquid and serum. For the diagnosis of bacterial ascites, the AUC of serum STREM-1 was 0.981 (95\% confidence interval $=0.962,0.999 ; \mathrm{P}<0.001$ ), which was greater than that of all the other indices. When the best cutoff point was set to 20.2, the sensitivity, specificity, positive predictive value and negative predictive value of serum sTREM-1 were 0.933, 0.955, 0.914, and 0.965, respectively. These results indicate that serum STREM-1 has higher diagnostic value for diagnosing bacterial ascites than the other three indices. Furthermore, the serum STREM-1 level was significantly decreased after treatment compared with that before treatment, indicating that this marker showed satisfying specificity to pre- and post-treatment bacterial ascites. Lemarié et al. compared the diagnostic value of STREM-1, calcitonin and CRP in septicemia and found that while all investigated indicators had certain value for the early diagnosis of septicemia, sTREM-1 had the highest sensitivity and specificity and that STREM-1 could be used for prognostication in cases of bacteremia [17].

However, our study showed that neither CRP nor STREM-1 had much clinical significance in differentiating the other three types of ascites. Yan and Zhan found that
sTREM-1 could be used as a rapid, convenient and safe method for differentiating parapneumonic pleural and tuberculous effusion in clinical practice, but our results contradict their findings [18]. The underlying reasons for this difference are presumably the inclusion of diseases with different complexity, sample sizes, inclusion and exclusion criteria between the studies. In addition, our finding may also confirm that each method for ascites differentiation has its own limitations and that the use of one method alone likely does not meet the requirements of clinical practice [19].

This study has the following limitations: it was conducted in a single center with a relatively small sample size. To overcome these drawbacks, multicentric studies with larger sample sizes need to be conducted.

In conclusion, STREM-1 may be more valuable for the diagnosis and differentiation of ascites in clinical practice than CRP and shows higher sensitivity and specificity, particularly in the diagnosis and prognostication of bacterial ascites.

Conflict of interest: Authors state no conflict of interest .

\section{References}

[1] Kuralay F, Tokgoz Z, Comlekci A. Diagnostic usefulness of tumor marker levels in pleural effusions of malignant and benign origin. Clin Chim Acta. 2000;300:45-55.

[2] Kleinberg L, Holth A, Fridman E, Schwartz I, Shih IeM, Davidson B. The diagnostic role of claudins inserous effusions. Am Clin Pathol. 2007;127:928-37.

[3] George MD, Østergaard M, Conaghan PG, Emery P, Baker DG, Baker JF. Obesity and rates of clinical remission and low MRI inflammation in rheumatoid arthritis. Ann Rheum Dis. 2017;76:1743-6.

[4] Lv JM, Wang MY. In vitro generation and bioactivity evaluation of C-reactive protein intermediate. PLoS One. 2018;13:e0198375.

[5] Bouchon A, Dietrich J, Colonna M. Cutting edge: inflammatory responses can be triggered by TREM-1, a novel receptor expressed on neutrophils and monocytes. J Immunol. 2000;164:4991-5.

[6] Bouchon A, Facehetti F, Weigand MA, Colonna M. TREM-1 amplifies in flammation and is a crucial mediator of septic shock. Nature. 2001;410:1103-7.

[7] Yuan Z, Syed MA, Panchal D, Joo M, Colonna M, Brantly M, et al. Triggering receptor expressed on myeloid cells 1 (TREM-1)mediated Bcl-2 induction prolongs macrophage survival. J Biol Chem. 2014;289:15118-29.

[8] Oudhuis GJ, Beuving J, Bergmans D, Stobberingh EE, ten Velde G, Linssen CF, et al. Soluble Triggering Receptor Expressed on Myeloid cells- 1 in bronchoalveolar lavage fluid is not predictive for ventilator-associated pneumonia. Intensive Care Med. 2009;35:1265-70. 
[9] Siranovic M, Kovac J, Gopcevic S, Kelecić M, Kovac N, Rode B, et al. Human soluble TREM-1: lung and serum levels in patients with bacterial ventilator associat-ed pneumonia. Acta Clin Croat. 2011;50:345-9.

[10] Phua J, Koay ES, Zhang D, Tai LK, Boo XL, Lim KC, et al. Soluble triggering receptor expressed on myeloid cells-1 in acute respiratory infections. Eur Respir J. 2006;28:695-702.

[11] Motherby H, Nadjari B, Friegel P, Kohaus J, Ramp U, Böcking A. Diagnostic accuracy of effusion cytology. Diagnostic Cytopathology. 1999;20:350-7.

[12] Gordon FD. Ascites. Clin Liver Dis. 2012;16:285-99.

[13] Yeh ET, Anderson HV, Pasceri V, WillersoN JT. C-reactive protein:linking inflammation to cardiovascular complications. Circulation. 2001;104:974-5.

[14] Prasad K. C-reactive protein and cardiovascular disease. Inter Angiol. 2003;12:1-12.

[15] Liao R, Liu Z, Wei S, Xu F, Chen Z, Gong J. Triggering receptor in myeloid cells (TREM-1) specific expression in peripheral blood mononuclear cells of sepsis patients with acute cholangitis. Inflammation. 2009;32:182-90.

[16] Hommes TJ, Hoogendijk AJ, Dessing MC, Van't Veer C, Florquin $\mathrm{S}$, Colonna $\mathrm{M}$, et al. Triggering receptor expressed on myeloid cells-1 (TREM-1) improves host defence in pneumococcal pneumonia. J Pathol. 2014;233:357-67.

[17] Lemarié J, Barraud D, Gibot S. Host response biomarkers in sepsis: overview on STREM-1 detection. Methods Mol Biol. 2015;1237:225-39.

[18] Yan J, Zhan XQ. Study of STREM-1 in differential diagnosis of parapneumonic pleural effusion and tuberculous effusion. Medical Information. 2015;47:53-4 (in Chinese with English abstract).

[19] Wang DJ, Zhang XY, Jiang XF, Jiang LQ. The value of CRP and PFt determination in the differentiation of exudates and transudate in pleural and abdominal cavity. Practical Clinical Laboratory. 2011;12:4-7 (in Chinese with English abstract) 